\title{
Dynamical failure of Turing patterns
}

\author{
Alon Manor and Nadav M. Shnerb \\ Department of Physics, Bar-Ilan University, Ramat-Gan 52900 Israel
}

\begin{abstract}
The emergence of stable disordered patterns in reactive systems on a spatially homogenous substrate is studied in the context of vegetation patterns in the semi-arid climatic zone. It is shown that reaction-diffusion systems that allow for Turing instability may exhibit heterogeneous "glassy" steady state with no characteristic wavelength if the diffusion rate associated with the slow reactant is very small. Upon decreasing the diffusion constant of the slow reactant three phases are identified: strong diffusion yields a stable homogenous phase, intermediate diffusion supports Turing (crystal like) patterns while in the slow diffusion limit the glassy state is the generic stable solution. In this disordered phase the dynamics is of crucial importance, with strong differences between local and global initiation.
\end{abstract}

PACS numbers:

\section{INTRODUCTION}

Pattern formation in reactive systems is a well investigated research field since the pioneering work of Turing [1] half a century ago. The idea of diffusion induced instability has been found to be applicable in many systems, ranging from animals coat patterns [2] to chemical reactions [3] and was analyzed mathematically using variety of techniques [4]. Generically, the instability of the spatially homogenous state is attributed to the existence of two different diffusion rates associated with the different reactants, where the inhibitor diffusion constant is faster than that of the activator. The resulting patterns are crystal-like, with a typical wavelength that, in many cases, is close to the characteristic length scale of the linear instability.

Recently, the applicability of Turing's idea to vegetation patterns in the semi-arid climatic zone has been studied by many authors [5]. The dynamics of this class of ecological systems is governed by the competition of perennial biomass units (shrubs, trees) for common resource (water). Taking into account some kind of "positive feedback" mechanism (like the slower mobility of soil moisture in the vicinity of the biomass), the corresponding reaction-diffusion equations lead to a Turing-like instability that may correspond to the observed ordered patterns.

It should be noted, however, that the generic situation in the semi-arid zone is disordered vegetation patterns, as clearly seen in Figure (1), where the results of typical field observations are presented. Interestingly, these patterns are quite robust and may stay unchanged for a long time (even hundreds of years). This is a somewhat surprising feature, as one expects a disordered system to wander among many microscopic configurations with equivalent features. The best known examples of disordered, stable patterns are observed when a supercooled liquid fails to reach its crystalline phase and freezes into a glass. Hence, the term "glassy phase" or "reactive glass" is used here to describe this phenomenon, already known in the field of coupled lattice maps 7].

A non-Turing mechanism, based on the periodicity of the water supply (dry and humid seasons) has already been suggested [6] in order to model the spontaneous spatial segregation into disordered stable patterns. In this letter we intend to present another route to the glassy state, a dynamical failure of the patterning at low diffusion on a lattice [8]. This mechanism seems to be a generic property of many Turing systems; accordingly, the water-vegetation equations are used here only to exemplify these features and to gain some basic intuition about their origin. The main aspect emphasized here is the appearance of many stable spatially disordered solutions for nonlinear dynamics. Applications to (and discussion of) vegetation patterns will be presented elsewhere.

Our basic observation is that the glassy phase exists, in many autonomous Turing systems on a lattice, provided that the mobility of the slow reactant is taken to be small enough. In that case generic initial conditions flow into a stable disordered pattern, long range spatial correlations disappear and the number of stable solutions grows exponentially with the size of the system. The deterministic reaction-diffusion dynamics is thus similar to the behavior of a dissipative, low temperature glassy system, that flows in phase space to the "closest" free energy local minima.

The importance of the dynamics is related to another analogy with the physics of glasses. The crystallization process in supercooled liquids takes place via the growth of a single nucleus, and if the growth rate is much slower than the production rate of microscopic nuclei the system fails to display long range order. In our system an analogous distinction should be made between global and local initiation. Starting from a single localized "seed" the biomass spreads into the unstable region and forms an ordered structure. Global initiation from random state, on the other hand, yields glassy pattern as small nuclei with different order parameters fail to merge into a macroscopic Turing state. In the following the glassy phase emergence from global initiation is first discussed, while the ordered patterns associated with local initiation are considered later on. 

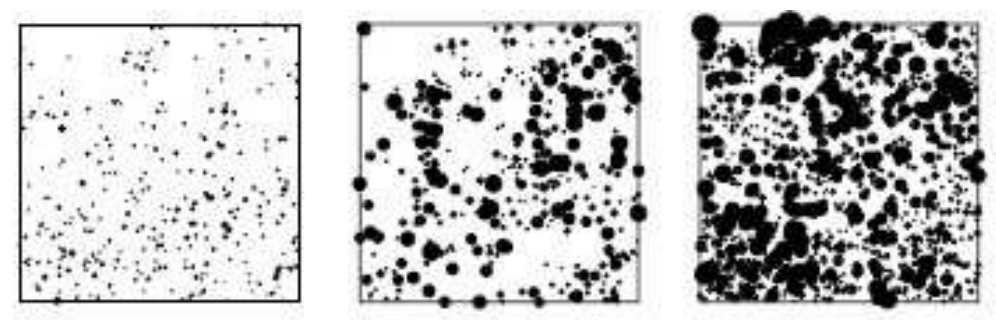

FIG. 1: Results of direct field measurements at three different locations along the precipitation gradient. The distribution of perennial shrubs (annual flora not included) is presented for an area of 100 square meters at each site. Each black spot presents a shrub and the size of a spot is proportional to the area of the canopy. Shrubs distribution on hillslopes has been taken at three sites representing mildly arid, semiarid, and subhumid climate conditions in Israel (see [6] for details).

\section{CHARACTERISTICS OF THE "GLASSY" PHASE}

Let us present a simple model for pattern formation in a biomass-water system. Denoting the water density by $w$ and the biomass density by $b$, a simple, dimensionless set of reaction-diffusion equations is:

$$
\dot{w}=R-\lambda w b-w+D_{w} \nabla^{2} w \quad \dot{b}=w b-\mu(b) b+D_{b} \nabla^{2} b
$$

The first equation describes the water (or soil moisture) dynamics, with constant deposition (rain) $R$, inorganic losses due to percolation and evaporation $(-w)$, consumption of water by the biomass $(\lambda w b)$ and diffusion $D_{w}$. The second equation expresses the biomass time evolution: it decays at rate $\mu(b)$, grows upon water intake, and diffuses at a much smaller rate, $D_{b}<<D_{w}$.

This simple set of equations supports spatial segregation only if there is some "positive feedback" mechanism that facilitates growth (at a spatial location) in the presence of biomass. In fact, perennial flora should reach some critical biomass threshold in order to survive the dry season, and this feature may be implemented in smooth reaction-diffusion equations by assuming smaller decay rate for larger shrubs. In this paper the following functional dependence of the decay rate $\mu$ is assumed,

$$
\mu(b)=\mu_{0}+\frac{\mu_{1}}{b+1}
$$

but the only essential trait is the monotonic decrease of the death rate as a function of the biomass size. With (2), Eq. (11) admits a Turing instability, and below some critical value of $D_{b}$ ordered patterns emerge, as demonstrated in the right panel of Figure 2

What happens if $D_{b}$ is taken to be even smaller? According to the standard linear analysis around the homogenous fixed point one expects to see ordered patterns all the way down to $D_{b}=0$, but on a lattice one gets, instead, the result shown in the left panel of Figure 2 with no apparent order or characteristic length scale. We stress that these disordered patterns are, indeed, linearly stable, as implied by direct numerical diagonalization of the linearized evolution operator. Moreover, both global initiation from the nearly empty state (small "seeds" of random height) and from the homogenous solution (small random fluctuations around it) yield the same type of disordered steady state.

One may guess, thus, that for small $D_{b}$ the phase space admits many attractive fixed points, each corresponds to a different disordered configuration. If a generic initial condition lays in the basin of attraction of one of these points, the deterministic evolution determined by Eq. (II) simply takes the system to that stable disordered point. Explicit counting of the number of stable fixed points (for small systems) shows, in fact, exponential growth with the system size in the glassy regime, as indicated in Figure 3. This exponential growth reflects the number of possible combinations of local patches and, correspondingly, the absence of long range order in the steady solutions.

As $D_{b}$ decreases, thus, the system undergoes an order-disorder transition, characterized by the loss of long range order and by the exponential dependence of the number of stable solution on the system size. A detailed analysis of that transition is beyond the scope of this paper; here we present some qualitative results that may be considered as a rough measure of that transition.

As a basic indication for the loss of long range order, the normalized radial correlation function is plotted against the radius as $D_{b}$ changes [Figure 4] left panel]. While for large $D_{b}$ the correlations are long range, at small diffusion only the first peak (that corresponds to the effective competition length) survives and there is almost no structure beyond that length scale. The normalized height of the second peak of the radial correlation function is also plotted (right panel) against the biomass diffusion coefficient and is shown to decrease with $D_{b}$ (almost linearly) up to some 


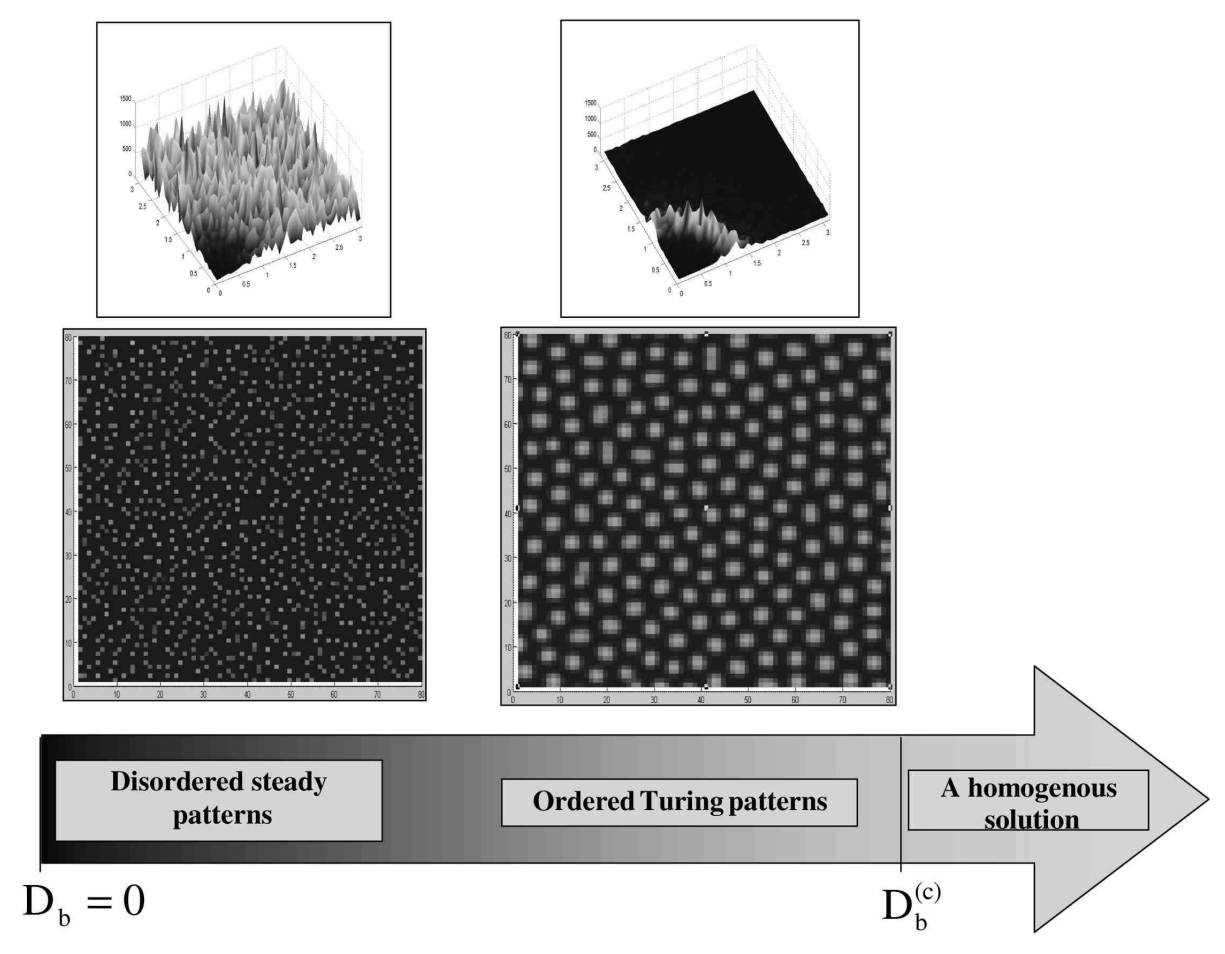

FIG. 2: Spatial patterns (lower panels) and their corresponding Fourier transform (upper panels) for different values of biomass diffusion. Numerical results of forward Euler integration of the reaction-diffusion equations (1-2) on an $80 \times 80$ sites grid with periodic boundary conditions are presented. In the right panel an ordered pattern is formed for $D_{b}=0.02$ and in the left panel a disordered pattern for $D_{b}=0.001$. The simulation parameters are $\mu_{0}=0.1, \mu_{1}=0.3, R=0.5, D_{w}=10$ and $\lambda=1.2$. Initial conditions are no water and a seed of biomass taken (at each site) from a square distribution between [0,0.01].

saturation at low values. One may identify the "critical" value of the diffusion constant with its value at the end of the plateau, where the system starts to respond to the increase of biomass spatial diffusion.

Another manifestation of the transition is the overlap of the steady state with the initial conditions. For a system dominated by a single attractive fixed point there is a complete memory loss, as all different initial states end up to be the same. As the number of attractive points increases it is plausible to assume that the phase space is divided into non-overlapping, compact basins of attraction, and a generic initiation flows to its "own" fixed point (in analogy with the diverging number of free energy local minima in glassy systems). In Figure 5 the overlap of the initial conditions and the final solution is plotted against the diffusion of the slow reactant, and the decrease of overlap as the system approaches the ordered Turing limit is clearly seen.

\section{III. "RICH GET RICHER" MECHANISM FOR DISORDERED SEGREGATION}

Our numerics suggests that this crossover from ordered to glassy patterns is a generic feature of lattice Turing systems: the same behavior has been observed for many different Turing systems we have examined (including the Gray-Scott and the Gierer-Meinhardt equations). Interestingly, our vegetation-water model also provides us with a simple intuitive argument that explains this transition.

The basic mechanism beyond the Turing instability is the "rich get richer" mechanism (Eq. 2) i.e., the fact that large shrubs are less effected by water shortage, so that even if small biomass units decay, large units still grow. 


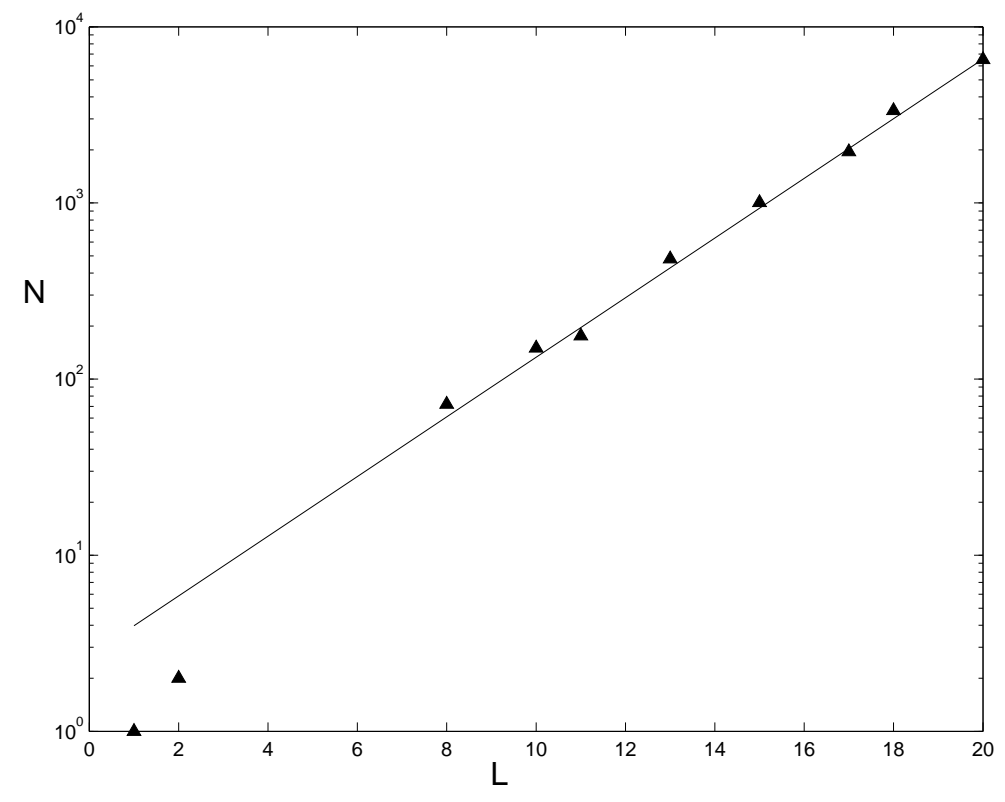

FIG. 3: Numerical counting of the number of stable solutions (N) as a function of the system size (L) for small, one dimensional samples where the sizes ranging from 1 to 20 sites. Solutions were found numerically using Newton-Raphson method with random sampling of the initial conditions phase space and their stability was checked by linear analysis. The numerical results (triangles) fit to an exponential growth with system size, $N \sim \exp (0.39 L)$, as indicated by the full line. (Boundary conditions are periodic, and all parameters are the same as in Fig. 2

Let us consider the case of $N$ independent biomass units using the same water resource, namely, the limit $D_{b} \rightarrow 0$, $D_{w} \rightarrow \infty$, of Eqs. (11). The biomass growth leads to a depletion of the water resource until, when $\mu(b)=w$ (for a specific shrub) it ceases to grow any more. However, at this resource level larger biomass units continue their development and the reduction of available resource, so the smaller shrubs start to wilt. As this process continues, only the largest biomass unit (at $t=0$ ) survives in the system. The mechanism of "rich get richer", thus, amplify small differences of the initial state to the level of "winner takes all", where only one unit survives the competition for common resource.

Turning back to the spatial model on a lattice with finite biomass and water diffusion, our numerical simulations indicate that the same logic may hold for small $D_{b}$ and large $D_{w}$. One may interpret these numerical results in view of the above argument: finite (but large) water diffusion leads to spatial segregation of the surface into large patches of competing biomass units, and the same "winner takes all" mechanism holds within each patch. For very small biomass diffusion each of these patches is still dominated by one large shrub, and the growth is so fast that the initial conditions dictate the final state of the system. The situation is close to the case of random sequential adsorption [9], where the system fails dynamically to reach its optimal filling. Larger biomass diffusion corresponds to slower takeover process, and the system dynamics admits stronger spatial correlations and allows for the emergence of Turing pattern.

\section{LOCAL INITIATION VS. GLOBAL INITIATION}

As emphasized above, the failure of the system to produce a Turing pattern is dynamical, as many localized excitations fail to merge into an ordered phase. This leads to the appearance of strong differences between different types of initial conditions. The results presented in the last sections are typical to the case of global initiation, where a homogeneous state is perturbed randomly at each site. If, on the other hand, there is a single "grain" (one perturbation with compact support) that grows and invades the unstable region, the system flows to the ordered phase even if the diffusion coefficient of the biomass is taken to be arbitrarily small (and the invasion of the stable solution into the unstable state becomes very slow). Comparing Figure (6) and the low $D_{b}$ pattern in Figure (2), the differences between local and global initiation are clearly recognized. 

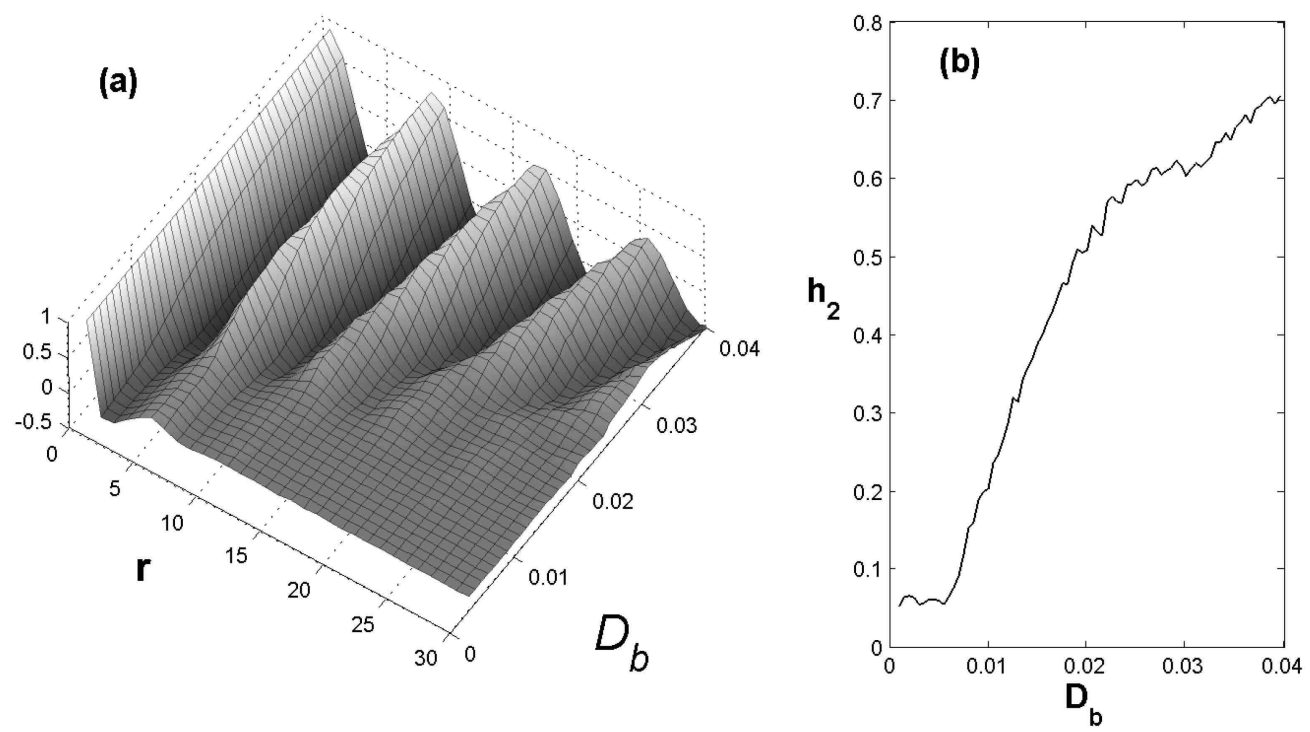

FIG. 4: (a) The correlation function $g(r)$ (in one dimension) is plotted against $r$ for different values of $D_{b}$, where all other model parameters are the same as in Figure 2 Clearly, there is a decay of the spatial correlation function as $D_{b}$ approaches zero. In the right panel (b) the second peak's hight, $h_{2}$, is plotted against $D_{b}$.

\section{CONCLUSIONS}

This paper deals with the possibility of dynamical failure in the process of development of Turing type patterns. This possibility has been demonstrated for the case of water-vegetation system on a lattice, where the biomass diffusion is small, such that its effect fails to compensate the faster "rich get richer" mechanism. The resulting patterns are disordered and robust without a characteristic length scale, and are strongly correlated to the random initial conditions. A Turing pattern does appear, for the same set of parameters, if the system is initiated locally, as the "rich get richer" ordering of shrubs according to their size coincides with the spatial ordering.

Although the numerical experiments presented here are only for the specific model of resource competition, the argument beyond the dynamical failure is generic, and one expects to see similar behavior in other Turing systems. For a system on finite lattice, at least, it is plausible to expect different localized excitations that (if the coupling between different points is not strong enough) fail to merge into one global pattern.

In analogy with coupled lattice maps [7], one may expect the glassy phase to disappear at the continuum limit (i.e., where the lattice size approaches zero). Note, however, that in any reactive system some degree of spatial discretization is dictated by the discrete nature of the reactants. In our case, for example, the discrete nature of a single shrub yields a basic length scale that corresponds to the minimal distance between the mother shrub and its propagule. Accordingly, the low diffusion glassy phase may be a feature of other realistic Turing system in the dilute limit, where the discrete nature of the reactants becomes important. 


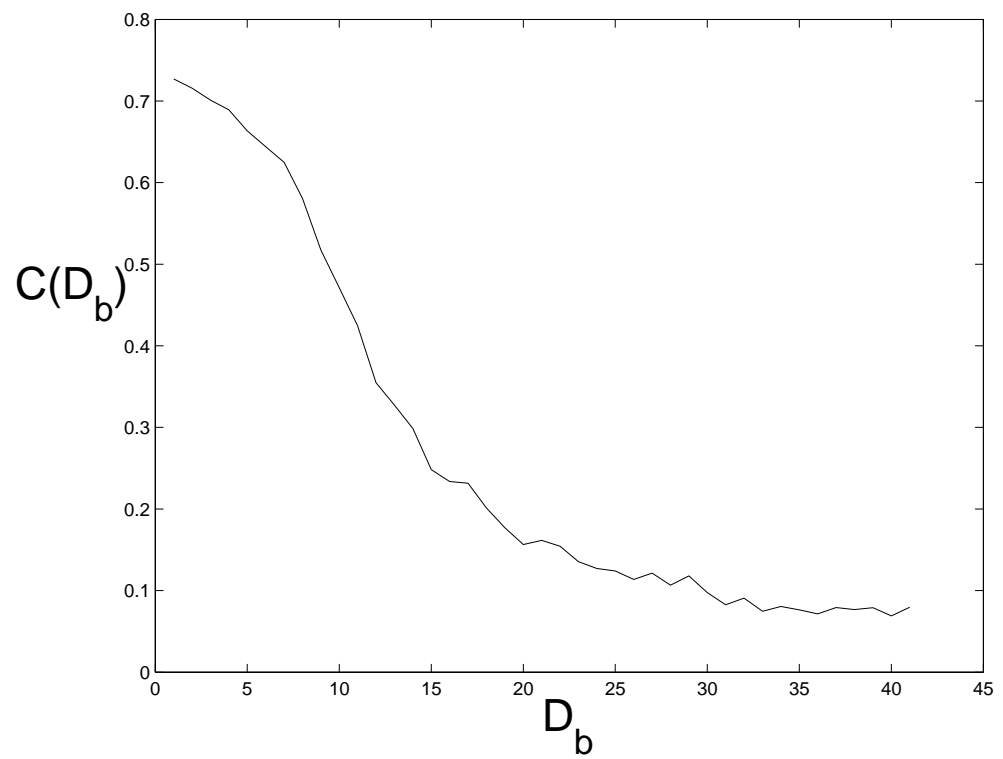

FIG. 5: The overlap $C\left(D_{b}\right)$ between the initial conditions and the resulting pattern is presented for different $D_{b}$ 's. With the same model parameters and initial conditions used above, the one dimensional steady state configuration shows much more overlap in the glassy phase than in the Turing phase. The initial conditions were mapped to a binary sequence (any local maxima is one, other points are zero) and the $L_{2}$ overlap of this vector with the final state is shown.

\section{Acknowledgments}

The authors thank David Kessler and Philip Maini for helpful discussion and comments. This work was supported by the Israeli Science Foundation, grant no. 281/03 and by Yeshaya Horowitz Fellowship..

[1] A. M. Turing, Phil. Trans. Roy. Soc. B 23737 (1952).

[2] See, e.g. J.D. Murray, Mathematical Biology (Springer-Verlag, New-York, 1993).

[3] V. Castets, E. Dulos, J. Boissonade and P. De Kepper, Phys. Rev. Lett., 64, 2953 (1990).

[4] M. C. Cross and P. Hohenberg, Rev. Mod. Phys. 65, 851 (1993); P.K. Maini et. al., J. Chem. Soc. Faraday T rans. 93, 3601 (1997).

[5] J. B. Wilson and A. D.Q. Agnew, Adv. Ecol. Res. 23, 263 (1992); R. Lefever and O. Lejeune, Bull. Math. Biol. 59, 263 (1997); J. von Hardenberg, E. Meron, S. Shachak, and Y. Zarmi, Phys. Rev. Lett. 87, 198101 (2001).

[6] N. M. Shnerb, P. Sarah, H. Lavee, and S. Solomon, Phys. Rev. Lett. 90, 038101 (2003).

[7] K. Kaneko, Physica (Amsterdam) 34D, 1 (1989); J. Kockelkoren, A. Lemaitre and H. Chate, Physica A 288, 326 (2000).

[8] Pattern formation and Turing instability have been recently demonstrated for a Belousov-Zhabotinsky system dispersed in water droplets of a reverse AOT microemulsion, see V. K. Vanag and I.R. Epstein, Phys. Rev. Lett. 87, 228301 (2001). The reaction takes place on diffusivly coupled, discrete patches; one may expect a transition to the glassy phase if the typical time associated with the migration of the slow reactant molecule between neighboring droplets is large enough.

[9] See, e.g., J. Talbot, G. Tarjus, P. R.Van-Tassel, and P.Viot, Colloids Surf. A, 165, 287 (2000), and references therein. 


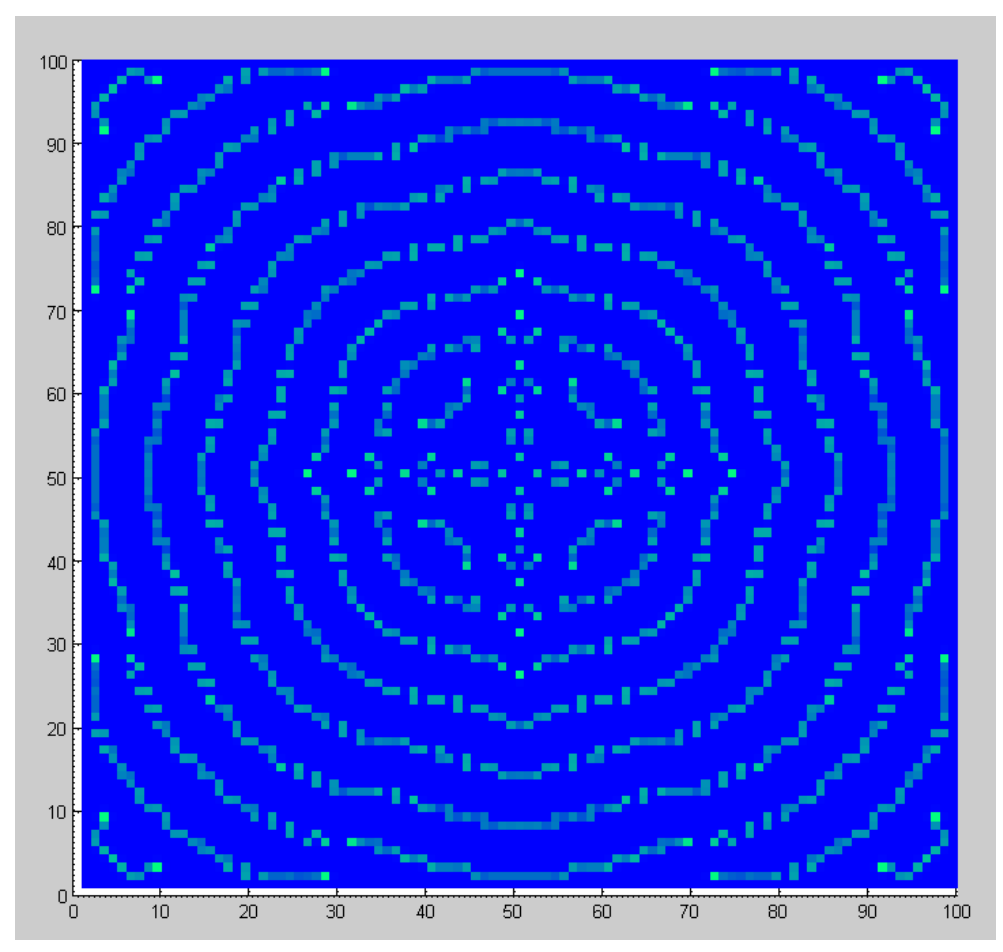

FIG. 6: Numerical results for local initiation in the glassy regime. Here, the case $D_{b}=0.002$ is evaluated where the initial conditions are only one seed in the middle of the sample, where all other parameters are the same as in Figure 2 The deviations from exact polar symmetry are due to the effect of the underlying square lattice. 